ARTICLE

\title{
Multi-Scale Turbulence Simulation in Magnetic Fusion Plasma
}

\author{
Jiquan LI ${ }^{1, *}$, Kenji IMADERA ${ }^{1}$, Paul HILSCHER ${ }^{1}$, \\ Yasuaki KISHIMOTO ${ }^{1}$ and Zhengxiong $\mathrm{WANG}^{2}$ \\ ${ }^{1}$ Graduate School of Energy Science, Kyoto University, Uji, Kyoto, Japan \\ ${ }^{2}$ School of Physics and Optoelectronic Technology, Dalian University of Technology, Dalian, China
}

\begin{abstract}
Complex nonlinear interaction of multi-mode multi-scale electromagnetic (EM) turbulence in magnetic confinement fusion (MCF) plasmas is explored based on large-scale direct simulations. As a practical step towards a full-scale turbulence simulation, two numerical approaches suitable for different spectral regime are proposed with an emphasis on the interaction mechanism among different fluctuations and structures. A gyrofluid model is employed to simulate the nonlinear evolution of mixed resistive MHD and micro-turbulence at ion-scale, which may clarify the energy-exchange mechanism between electrostatic and EM fluctuations. As a result, a magnetic island seesaw oscillation and a short wavelength ion-scale drift wave are observed in multi-scale turbulence simulations with different equilibrium magnetic field. The underlying mechanism for the former one is identified as a cross-scale dynamo generation by the micro-turbulence through an elucidation of minimal modeling analysis. Meanwhile the latter one is induced by a wide magnetic island under the ion temperature island collapse. On the other hand, a strategic gyrokinetic simulation plan is presented to explore the micro-turbulence and transport property with both adiabatic kinetic ion and electron responses. A gyrokinetic Vlasov code is advanced to simulate the nonlinear interaction between ion and electron scale turbulence aiming at understanding the long time behaviors of turbulent transport in burning plasmas. The code parallelization is also introduced briefly.
\end{abstract}

KEYWORDS: multi-scale plasma turbulence, MHD and dynamo, micro-turbulence and zonal flows, gyro-fluid and gyrokinetic simulation

\section{Introduction}

Magnetic confinement fusion (MCF) is highly expected as one of promising approaches for supplying future energy demand. A milestone event in the progress is the starting construction of the ITER (International Thermonuclear Experimental Reactor) device recently in France. ${ }^{1)}$ However, the physics study on improving the plasma confinement performance is still a crucial issue for present tokamak and the future ITER although the ITER is expected to take the fusion to the stage of industrial application. An important aspect in ITER physics is the understanding of complex turbulence property in burning plasma to reduce the turbulent transport. $\left.{ }^{2}\right)$ The study of plasma turbulence and anomalous transport is always a central topic in the field. This is because fusion plasma is generally subject to various instabilities at differing spatio-temporal scales due to varied magnetic configurations and plasma profiles. For example, the fluctuation in tokamak plasma is characterized by macro-scale MHD and micro-scale drift wave turbulence. The theorists and experimentalists have been struggling such instabilities to control plasma and improve confinement for a time with the aid of massively parallel simulations. As the progress of parallel computing, large-scale simulation using tens of thousands of CPUs becomes feasible to cover cross-scale turbulence due to different modes. This work aims at proposing an attempt to perform multi-mode multi-

*Corresponding author, E-mail:1ijq@energy.kyoto-u.ac.jp scale turbulence simulation with different electromagnetic (EM) features in Tokamak plasmas.

In MCF plasmas, the modern gyrokinetic theory ${ }^{3)}$ is generally employed to develop first principle particle code using particle-in-cell method or continuum (Vlasov) code with advanced computational fluid dynamics (CFD) schemes. Such large-scale simulations are promoted by the high- performance parallelization. An amount of gyrokinetic codes have been applying in present tokamak and stellarator towards the ITER. ${ }^{4)}$ On the other hand, the fluid (or gyrofluid) approach in 3-dimensional (3D) configuration is still helpful and has advantages to clarify the dominant physical processes and to scan the parametric dependence in a long time simulation, particularly for macro-scale MHD fluctuations. Hence, this work proposes both gyrofluid and gyrokinetic approaches to explore the cross-scale problem involving the MHD island dynamics and micro-turbulence. The attention is focused on the nonlinear interaction mechanism between different modes with different scales and EM features. The development of the gyrokinetic Vlasov code as a strategic target is briefly introduced.

The reminder of this paper is organized as follows. In Section II, the multi-scale turbulence problem associated with the simulation approach in MCF plasmas is described. The gyrofluid simulation on mixed MHD and micro-turbulence at ion-scale is performed in Section III. The results are presented with the emphasis on the new dynamics due to cross-scale interaction. In Section IV, the develop- 
ment of a gyrokinetic code to simulate both kinetic ion and electron responses on equal foot is introduced aiming at chasing the next generation exa-scale simulation in the study of long-time dynamics of multi-scale turbulence and transport in MCF plasmas. Finally, the work is summarized in Section V.

\section{Multi-Scale Turbulence Problem and the Prac- tical Simulation Models in MCF Plasmas}

\section{Multi-Scale Turbulence Problem in MCF Plasmas}

In tokamak and stellarator plasmas, the fluctuations are highly anisotropic in spatio-temporal spectra due to the Lorentz force on the charged particles. ${ }^{5)}$ The gyro-motion of charged particles is much faster than the diamagnetic drift due to the profile inhomogeneity. These low frequency diamagnetic drift and the high frequency gyro-motion may be the most fundamental multi-scale problems in MCF plasmas. Such two-scale separation provides the basic hypothesis of the gyrokinetic theory, in which the fast gyro-motion is removed through gyro-averaging approximation. ${ }^{3)}$ For the low frequency fluctuations, on one hand, the multi-scale (mainly two-scale) problem with parallel long wavelength and perpendicular short wavelength described by the gyrokinetic model has been approximately solved through the so-called ballooning representation in a sheared geometry. ${ }^{6}$ On the other hand, the multi-scale problem due to ion and electron responses on equal footing along the perpendicular direction should be solved by the direct simulation. Hence the multi-scale problem invoked here is on the nonlinear turbulence interaction in a wide spatial scale covering the electron and ion gyro-radius as well as the device size scale and in a broad time scale cross the electron and ion diamagnetic drift time and the Alfven time of magnetic fluctuations. The multi-scale physics is governed by both non-adiabatic responses of electrons and ions as well as the magnetic dynamics through the nonlinear coupling. The fluctuation at each scale may be represented by some typical eigenmodes, as schematically pictured in Fig. 1 in tokamak plasmas. Each mode is linearly or nonlinearly driven by individual driving force and nonlinearly connected with others through mode coupling. Note that the spatio-temporal scale widely covers several orders in spectral range so that the analytical solution of each fluctuation is almost impracticably obtained at present if the nonlinear interaction processes in whole spectrum are fully involved. A new theory framework of multi-scale plasma turbulence is under the construction.

Massively parallel plasma simulation has become an indispensable method in turbulence study and the relevant work has been running for years. ${ }^{7)}$ Based on the theoretical description of plasma behavior, the simulation methodology is categorized as fluid and kinetic simulations. The former one generally includes the MHD turbulence simulation. The first principle simulation in MCF plasmas is based on modern gyrokinetic theory ${ }^{3)}$ employing particle or Vlasov method. In gyrokinetic simulation, the particle motion in a turbulent environment is precisely solved in time. The feedback to the fluctuation is evaluated through statistical average procedure. Hence it suffers from onerous calculation

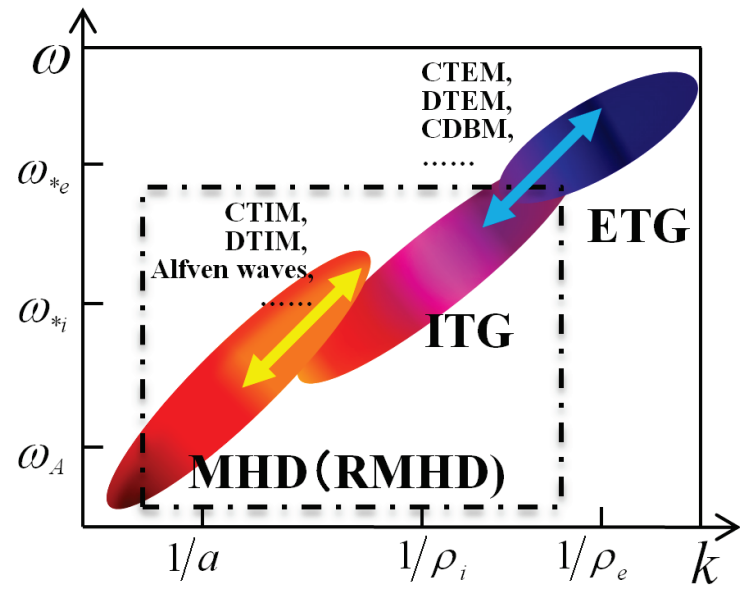

Fig. 1 Schematic system of multi-scale turbulence in MCF plasmas. Here $a$ is device size, $\rho_{i, e}$ is ion or electron gyroradius, $\omega_{* i, e}$ is the diamagnetic drift frequency of ion or electron, $\omega_{A}$ is the Alfven frequency. The abbreviated forms are expressed as follows: MHD/RMHD-magnetohydrodynamics (resistive MHD), ITG/ETG-ion/electron temperature gradient, CTIM/DTIM (CTEM/DTEM)-collisionless/dissiptive trapped ion/electron mode, CDBM-current diffusion ballooning mode.

and huge CPU time while the reliable results are generally respected. The advantage of the fluid model is renowned. It has been extensively shown that the fluid simulation has not only revealed an amount of physics mechanisms through simplified modeling and sophisticated treatment, but it may also be more appropriate to perform long time simulation of relevant physics processes, which is required in future fusion reactor plasma including the ITER burning plasma.

Multi-scale turbulence problem in MCF plasma is extremely complicated due to various instabilities distributed in wide spatio-temporal spectra. It seems still unfeasible at present to perform a full-scale turbulence simulation covering all MHD activities, ion- and electron-scale microfluctuations as well as the transport dynamics. While many efforts are being made to consistently involve nonadiabatic ion and electron responses over a transport time scale in a first principle gyrokinetic simulation, various simplified models that emphasize important physics processes in a relatively narrow spectral regime are also being proposed as a practical step. Two typical cases are feasible. One case is the multi-scale combination with resistive MHD (RMHD) and ion-scale micro-fluctuation, namely RMHD+ITG in Fig. 1, in which the electron inertia effect is ignored. This model aims at exploring the nonlinear interaction between magnetic island dynamics and ion-scale turbulence with zonal flows (ZFs). Another model tends to involve kinetic ion and electron responses on equal foot as the typical system ITG+ETG as illustrated in Fig. 1. The basic difference from the RMHD+ ITG model is the retaining of full electron inertia effect so that the micro-scale electron dynamics can be studied in the ITG+ETG model. In addition, the latter might be also applicable to simulate the collisionless magnetic reconnection with the full electron inertia effect, which will be considered in the future work. 


\section{Simplified Models of Multi-Scale Turbulence Simula-} tion in MCF Plasmas

(1) Gyrofluid Model for Multi-Scale RMHD+ITG Turbulence Simulation

MHD dynamics is generally a slow process on the Alfven time scale for the fluctuations with low mode number in low $\beta$ plasmas, which is much longer than the time scale typical of ion's and electron's dynamics. Hence it is hard to numerically incorporate full kinetic electron response in multiscale turbulence simulation with MHD fluctuation due to light electron mass. To investigate the MHD influence on micro-turbulence and transport, a simplified model mainly including essential MHD dynamics and ion-scale turbulence is principally feasible. Here the physics model is referred to as RMHD+ITG turbulence if a typical ion-drift wave ITG mode is sampled as an example. In this sense, the ITG turbulence should be typical of an EM fluctuation. In low $\beta$ plasmas, the ITG turbulence is characterized mainly by an electrostatic (ES) potential. Contrarily, a micro-scale Alfventype ITG fluctuation, namely the AITG ${ }^{8)}$ may dominate the multi-scale EM turbulence as the $\beta$ value increases. For these two cases, the interaction between MHD and ion-scale micro-turbulence may be inherently different. In addition, the former case may also become a test-bed to study the energy-exchange mechanism between multi-mode EM and ES fluctuations.

Numerically, the multi-scale RMHD+ITG turbulence is simulated using a gyro-Landau-fluid model, ${ }^{9)}$ or gyro-fluid model,${ }^{10)}$ in which two key kinetic effects of both FLR and Landau damping are approximately included besides the advantages of fluid model. The focus here is on the underlying interaction mechanism so that the dominant physics processes are essentially retained in the model. Most importantly, the gyrofluid model can capture the effect of MHD island evolution on the ion-scale turbulence through the gyrofluid closure, that is, the Landau damping is calculated along the perturbed magnetic field line. For simplicity, the FLR effect is still able to be replaced by the cross-field dissipation terms such as the hyper-viscosity at short wavelength regime. ${ }^{11,12)}$

(2) Gyrokinetic Model for Multi-Scale ITG+ETG Turbulence Simulation

Micro-turbulence is always one of most important topics in MCF plasma study since it is believed to be responsible for the anomalous transport and confinement performance. ${ }^{13)}$ Typical micro-instabilities at individual ion and electron scales have been investigated theoretically and numerically for years. In each case, a component with adiabatic response to the potential perturbation is assumed. To explore the mutual influence between them, a multi-scale ITG+ETG turbulence simulation including both kinetic ion and electron responses is necessary. Although either ITG or ETG turbulence can be simulated using gyrofluid model, precise ion FLR effect and closure relation for Landau damping are crucial in a wide spectrum. In addition, higher order gyrofluid moment equations are rather complicated so that the fluid-type model may lose the advantage. In this sense, gyrokinetic theory is directly employed aiming at the first principle simulation. Gyrokinetic model is in principle simple for multi-scale turbulence simulation as long as the ions and electrons are treated on equal foot. The kinetic ion and electron responses are physically linked through the Poisson's equation. However, the conservation law and the high accuracy scheme are strictly required for long time simulation with a wide spectrum. ${ }^{4)}$ Except for alternative particle method using PIC scheme, the Vlasov approach is employed in this work, in which the kinetic equation is directly solved through a multi-dimensional finite difference using high accuracy CFD scheme. Practically, an artificially reduced ion/electron mass ratio has to be assumed at present test. In addition, various approximations may be considered to focus the attention on the underlying interaction mechanism between ion and electron scale fluctuation.

\section{Gyrofluid Simulation of Multi-Scale RMHD+ITG Turbulence}

Aiming at understanding the interplay between multiscale MHD and ion-scale turbulence, a 5-field gyrofluid model is employed to perform multi-scale simulation. ${ }^{14)}$ In a normalized form for the ion-scale drift wave, the model describing the multi-scale EM turbulence consists of the nonlinear evolution equations of continuity (density $n$ ), ES potential $\phi$, parallel component of vector potential $A_{/ /}$ (usually denoted by magnetic flux $\psi=-A_{/ /}$), parallel ion velocity $v_{\| /}$, and ion temperature $T_{i}$, as follows

$$
\begin{aligned}
& d_{t} n=-\partial_{y} \phi-\nabla_{/ /} \mathcal{v}_{/ /}+\nabla_{/ /} j_{/ /}+D_{n} \nabla_{\perp}^{2} n, \\
& d_{t} \nabla_{\perp}^{2} \phi=\left(1+\eta_{i}\right) \partial_{y} \nabla_{\perp}^{2} \phi+\nabla_{/ /} j_{/ /}+\mu_{\perp} \nabla_{\perp}^{4} \phi, \\
& \beta \partial_{t} A_{/ /}=-\nabla_{/ /}(\phi-n)-\beta \partial_{y} A_{/ /}-\eta j_{/ /}, \\
& d_{t} v_{/ /}=-2 \nabla_{/ / n}-\nabla_{/ /} T_{i}+\beta\left(2+\eta_{i}\right) \partial_{y} A_{/ /}+\eta_{\perp} \nabla_{\perp}^{2} \mathcal{v}_{/ /}, \\
& d_{t} T_{i}=-\eta_{i} \partial_{y} \phi-\frac{2}{3} \nabla_{/ / \mathcal{v}_{/ /}}-\frac{2}{3} \sqrt{\frac{8}{\pi}}\left|\nabla_{/ /}\right| T_{i}+\chi_{T} \nabla_{\perp}^{2} T_{i} .
\end{aligned}
$$

with $j_{/ /}=-\nabla_{\perp}^{2} A_{/ /}, \quad \beta=8 \pi n_{0} T_{i 0} / B^{2}, \quad \eta_{i}=d \ln T_{i} / d \ln n$. The operators $d_{t}=\partial_{t}+\hat{e}_{z} \times \nabla_{\perp} \phi \cdot \nabla_{\perp}, \quad \nabla_{\perp}^{2}=\partial_{x}^{2}+\partial_{y}^{2}$ and $\nabla_{/ /}=\partial_{z}+\hat{e}_{z} \times \nabla\left(A_{/ / 0}+A_{/ /}\right) \cdot \nabla_{\perp}$ are expressed in slab geometry $\quad \vec{B}=B_{0} \hat{e}_{z}+B_{y} \hat{e}_{y}=B_{0} \hat{e}_{z}-\beta \nabla A_{/ / 0}(x) \times \hat{e}_{z} \quad$ with constant $B_{0}$. In this work, two different equilibrium models $B y 1 \propto \tanh \left(x / \lambda_{1}\right) / \cosh ^{2}\left(x / \lambda_{1}\right) \quad$ and $\quad B y 2 \propto \operatorname{arcsinh}\left(x / \lambda_{2}\right)$ are employed as shown in Fig. 2. Here $x$ is the distance deviated from the singular surface, $\lambda$ is the gradient length of axial equilibrium current. Note that near the singular surface, namely the rational surface $x=0$, both models are reduced to the usual slab model in tokamak plasmas. ${ }^{15)}$ The normalization is employed as follows

$$
\begin{aligned}
&\left(x / \rho_{i} ; y / \rho_{i} ; z / L_{n} ; v_{t i} t / L_{n}\right) \rightarrow(x ; y ; z ; t), \\
&\left(L_{n} / \rho_{i}\right)\left(\tilde{n} / n_{0} ; e \widetilde{\phi} / T_{i 0} ; \widetilde{v}_{\| /} / v_{t i} ; 2 e v_{t i} \widetilde{A}_{/ /} / \beta c T_{i 0} ; \widetilde{T}_{i} / T_{i 0}\right), \\
& \rightarrow\left(n ; \phi ; v_{/ /} ; A_{/ /} ; T_{i}\right)
\end{aligned}
$$

with $T_{i}=T_{e}, \quad \rho_{i}=v_{t i} / \omega_{c i}, \quad v_{t i}=\sqrt{T_{0} / m_{i}}, \quad \omega_{c i}=e B_{0} / m_{i} c$ and 


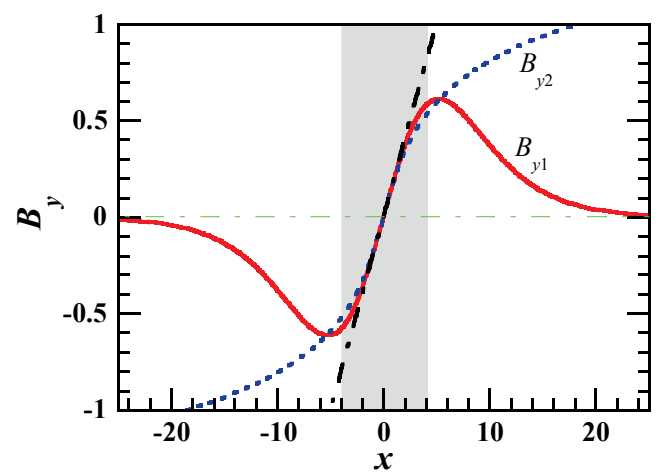

Fig. 2 Modeling equilibrium $B y 1 \propto \tanh \left(x / \lambda_{1}\right) / \cosh ^{2}\left(x / \lambda_{1}\right)$ and $B_{y 2} \propto \operatorname{arcsinh}\left(x / \lambda_{2}\right)$. The dot-dashed line corresponds to conventional sheared slab geometry.

$L_{n}^{-1}=d \ln n_{0} / d x$. Hence the corresponding frequency is in the unit of the diamagnetic drift frequency. In deriving these equations, the gyrofluid closure for Landau damping ${ }^{9)}$ and classical cross-field dissipations $\left(D_{n}, \mu_{\perp}, \eta_{\perp}, \chi_{T}\right)$ for short wave components ${ }^{11,12)}$ are used. This model may be regarded as an integrated system describing the resistive MHD and micro-scale ITG modes. It can be explicitly shown by two reduced cases. At the ES limit, usual 3-field ITG model is derived with adiabatic electron response. ${ }^{15)}$ Contrarily, ignoring the drift wave effect can reduce to the conventional incompressible 2-field MHD model described by Eqs. (2) and (3). ${ }^{16)}$ The multi-scale turbulence may be jointly controlled by two key parameters: $\eta_{i}$ and the resistivity $\eta$.

The simulation can be performed using an initial value code ${ }^{14)}$ which solves the nonlinear evolution equations (1)-(5) in a 2-dimensional $(x, y)$ plane perpendicular to the equilibrium magnetic field along the $z$ direction. The code is designed for solving a general 3-dimensional problem. Fourier transformation in $y$ and $z$ directions for any perturbed physical quantity is expressed as

$$
f(x, y, z, t) \sim \sum_{m, n} f_{m, n}(x, t) \exp \left(i 2 \pi m y / L_{y}-i 2 \pi n / L_{z}\right)
$$

with corresponding wave numbers $k_{y}=2 \pi m / L_{y}$, $k_{z}=2 \pi n / L_{z}$ and $k_{/ /}=2 \pi n / L_{z}-2 \pi m \hat{x} x / L_{y}$. An implicit finite difference scheme (namely so-called Crank-Nicholson scheme) for $x$ variable is used with fixed boundary conditions at $x= \pm L_{x} / 2$. Radial domain $L_{x}=100$ is taken, which is much larger than the width of singular layer. The domain in $y$ direction takes $L_{y}=20 \pi$ so that the longest wavelength component $k_{y}=0.1$ corresponds to the usual $m=1$ MHD mode in a toroidal or cylindrical plasma. The second or fourth order Runge-Kutta method is applied for the time advance. The time step is adjusted under the Courant condition. Reference parameter setting except for the key parameter pair $\left(\eta_{i}, \eta\right)$ in mixed-scale turbulence simulations is fixed as $\beta=0.01, \hat{s}=0.2, \lambda_{1}=25, \lambda_{2}=5$, $D_{n}=\mu_{\perp}=\eta_{\perp}=\chi_{T}=0.01$.

Linear simulation shows that two instabilities can be excited simultaneously in a whole spectrum by properly

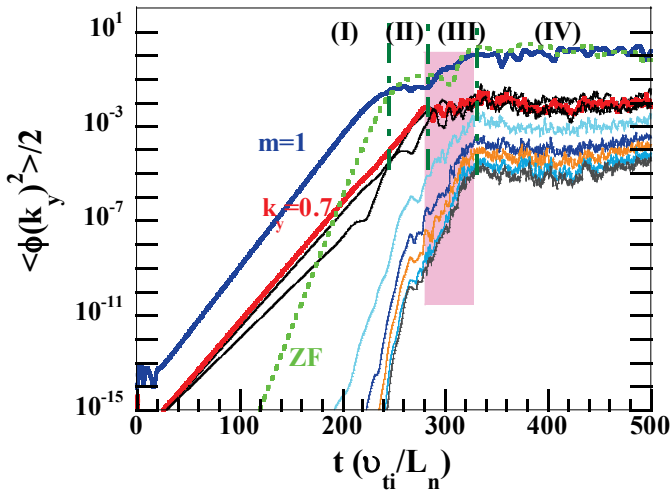

Fig. 3 Time history of kinetic energy in multi-scale MHD and ITG turbulence. $\eta_{i}=2.0, \eta=5 \times 10^{-4}, \lambda_{1}=25$.

choosing $\left(\eta_{i}, \eta\right)$. The ITG fluctuation is located around the peak $k_{y} \rho_{i} \sim 0.6$ corresponding to usual ion-drift ITG mode. Meanwhile, the low mode number MHD fluctuations are unstable for $m \leq 2$. The MHD-dominated EM fluctuation tends to be typical of the drift tearing mode due to the diamagnetic drift effect. The magnetic island tends to propagate along electron diamagnetic drift direction since the normalized real frequency is positive for most unstable MHD. Hence, this modeling can be employed to investigate the direct nonlinear interaction between MHD mode and micro-instability with different driving forces.

\section{Magnetic Island Dynamics Nonlinearly Influenced by Micro-Turbulence}

First the equilibrium with $B_{y 1} \propto \tanh \left(x / \lambda_{1}\right) / \cosh ^{2}\left(x / \lambda_{1}\right)$ is considered. The sheared poloidal magnetic field tends to be weak at edge region far from the singular surface as shown in Fig. 2, it is expected the magnetic island dynamics may be easily influenced by micro-turbulence. The direct gyrofluid simulations are mainly performed with unstable MHD and different ITG instabilities. In these direct simulations, the zonal components for all variables including the zonal density and temperature are consistently kept except for some test cases in which the zonal flows are artificially excluded. All simulations show a common evolution history with four phases as illustrated in Fig. $\mathbf{3}$ as an example. Unstable MHD with $m=1$ and ITG instability around $k_{y}=0.7$ are linearly excited at Phase I. In this phase, the stable or weaker instability may be nonlinearly accelerated by the stronger one if the growth rates are different. This process accompanying with the ZF stabilization leads to an initial saturation at a lower level (Phase II). Afterwards, complex nonlinear interactions occur with the growth of the zonal modes (ZFs; zonal fields and pressures), the turbulent fluctuations increase again (Phase III), finally saturate at a higher level in quasi-steady state (Phase IV). In Phase III, the growth rate of the $m=1$ component is roughly estimated to be the same as that in Phase I. Most importantly, a pronounced magnetic island oscillation is commonly observed in the case with a full reconnection (namely, with larger tearing instability parameter $\left.\Delta^{\prime}[17]\right)$, showing a dynamic quasi-steady state in Phase IV. An oscillatory ZF with 


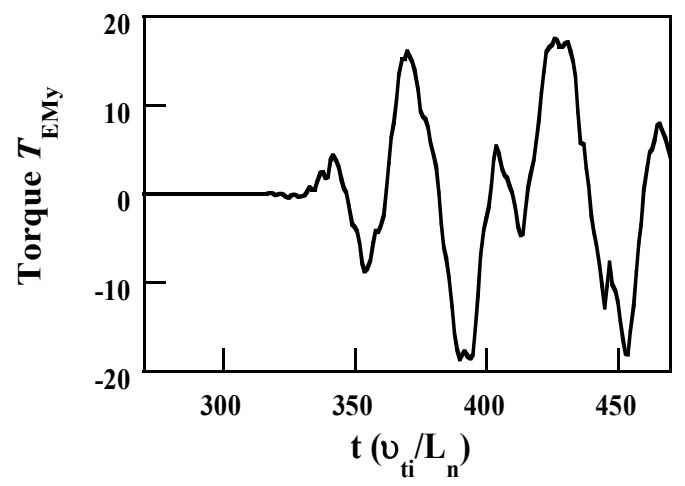

Fig. 4 Time history of averaged EM torque in the simulation of Fig. 3.

finite frequency also occurs. The island oscillation is visualized by a movie of island evolution, and much more clearly, by snapshots of the dominant flux component $m=1$. The island oscillation occurs pivoting along the singular surface like a seesaw (referred to hereafter as an island seesaw). The averaged EM torque ${ }^{18)}$

$$
T_{E M y} \hat{z}=\iint_{x y} x \hat{x} \times(\vec{j} \times \vec{B})_{y} d x d y / L_{x} L_{y}
$$

exerted on the island by the fluctuating EM force in the $y$ direction increases dramatically and tends to oscillate in time in the quasi-steady state, as shown in Fig. 4, synchronizing with the island seesaw. The EM torque and seesaw amplitude increase with increasing $\eta_{i}$. For smaller $\Delta^{\prime}$, the magnetic reconnection also occurs, but the island is saturated at small width, so the magnetic island remains static regardless of the ITG intensity.

A minimal model, ${ }^{19)}$ which mainly includes reduced MHD and an individually evolving ITG eigenmode, is proposed to capture the dominant nonlinear interaction between micro-scale ITG fluctuation and the MHD island. It is found that a cross-scale dynamo action is induced by ITG flows, which depends on the radial parity. The modeling analyses can well reproduce the main features of the island seesaw observed in direct simulation so that the minimal model may be used to investigate the energy-exchange channel between ES and EM plasma fluctuations.

\section{Short Wavelength ITG Excitation Nonlinearly In- duced by a Magnetic Island}

In tokamak plasma, local equilibrium near the low $q$ rational surface is approximated as $B y 2 \propto \operatorname{arcsinh}\left(x / \lambda_{2}\right)$. In such situation, the magnetic island is likely saturated with finite width. Numerical experiment is designed to explore the micro-turbulence behavior in a turbulent environment with dynamical magnetic island, likely in the Rutherford stage of the nonlinear tearing mode. The evolution of the multi-scale turbulence is similar to that in Fig. 3 but a sudden excitation of short wavelength ES potentials with almost an identical growth rate in the quasi-steady state as shown in Fig. 5, while the magnetic island is still evolving very slowly. The spectral evolution of the new instability is analyzed as shown in Fig. 6, showing a large spectral hump around $k_{y} \rho_{i} \geq 1$ which deviates from usual ITG spectrum. The

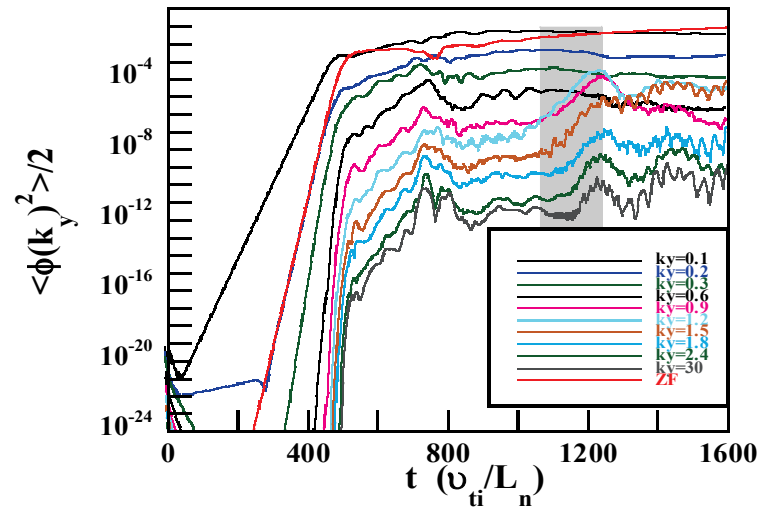

Fig. 5 Time history of kinetic energy in multi-scale MHD and ITG turbulence. $\eta_{i}=2.0, \eta=5 \times 10^{-4}, \lambda_{2}=5$.

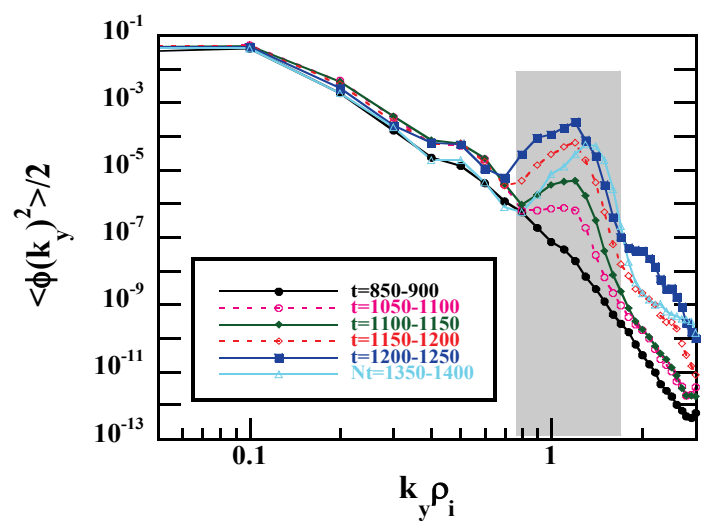

Fig. 6 Spectral evolution of new instability in the quasi- steady state of multi-scale MHD and ITG turbulence in Fig. 5.

fluctuation is characterized by weak magnetic component and propagating in the ion diamagnetic drift direction surrounding the magnetic island. The growth rate also depends on the initial equilibrium $\eta_{i}$ although the temperature profile is modified by the zonal temperature through the quasi-linear flattening effect. These features suggest a secondary short wavelength ITG instability. Furthermore, it is observed that the plasma frozen-in condition is fairly satisfied in the early transient phase of quasi-steady-state as the magnetic island grows very slowly. As the dynamical nonlinear interaction continues among MHD and ITG fluctuations as well as all zonal modes, the energy of $m=1$ component of the ion temperature fluctuation decreases sharply so that the ion temperature island collapses. The density island as well as the magnetic island still survives following the constraint of the frozen-in condition. Afterwards, the short wavelength ITG fluctuations exponentially grow up. The sequence of the events shows that the ion temperature island collapse is a precondition of the secondary excitation of the short wavelength instability.

It is generally expected that the profile flattening occurs inside the magnetic island so that the micro-instability may be stabilized. ${ }^{20,21)}$ On the other hand, recently a preliminary linear analysis under a fixed ion temperature profile shows 
that a wide magnetic island may enhance ITG instability. ${ }^{22)}$ Based on such arguments, it may be understood that the short wavelength ITG instability is induced by a growing magnetic island once the collapse of the ion temperature island occurs. The temperature island collapse may depend on the heat transport property along and perpendicular to the perturbed magnetic field directions. The physical mechanism remains to be understood and will be reported later.

\section{Gyrokinetic Simulation of Multi-Scale ITG+ETG Turbulence}

The modern gyrokinetic theory, which describes the gyro-averaged distribution function of particles, consists of the guiding-center and gyro-center transforms based on the Hamiltonian formalism through the Lie perturbation analysis. ${ }^{3,4)}$ It enables mathematically unambiguous and rigorous treatment of collisionless turbulent dynamics with keeping the particle and energy conservation, which are important to describe the long-time turbulent transport process and perform the first principle simulation. On the other hand, the high performance parallel computing allows one to simulate more realistic physical processes including multi-scale micro-turbulence interaction. ${ }^{23,24)}$ Here we employ the Vlasov approach, which directly solves the guiding center distribution function with keeping the FLR effects by discretizing the phase space, to simulate the micro- turbulence evolution cross the ion and electron scales. The gyrokinetic simulation model for the multi-scale ES micro-turbulence is governed in slab geometry by the gyrokinetic equation of the particle distribution function $F$ in 5-dimensional phase space $\left(\vec{X}, \mu, v_{/ /}\right)$in non-canonical gyro-center coordinate

$$
\frac{\partial F_{\alpha}}{\partial t}+\left(v_{/ /} \hat{b}+\frac{c}{B_{0}} \hat{b} \times \nabla\langle\Phi\rangle_{\phi}\right) \cdot \frac{\partial F_{\alpha}}{\partial \vec{X}}-\frac{q_{\alpha}}{m_{\alpha}} \hat{b} \cdot \nabla\langle\Phi\rangle_{\phi} \frac{\partial F_{\alpha}}{\partial v_{\|}}=0,
$$

Here $\langle\Phi\rangle_{\phi}=(2 \pi)^{-1} \oint \Phi d \phi=\sum_{\vec{k}} \Phi(\vec{k}) J_{0}\left(k_{\perp} \rho_{\alpha}\right) \exp (i \vec{k} \cdot \vec{X})$ is the gyroaveraging operator, $\hat{b}=\vec{B} / B_{0}$ is the unit vector, $\phi$ is the gyro-phase angle defined as $\phi=\tan ^{-1}\left(\hat{e}_{1} \cdot \vec{v} / \hat{e}_{2} \cdot \vec{v}\right)$ with arbitrary orthogonal unit vectors $\hat{e}_{1}$ and $\hat{e}_{2}$ in the plane perpendicular to $\hat{b}$. The ES potential $\Phi$ is determined by the gyrokinetic Poisson equation

$$
\begin{aligned}
& -\nabla^{2} \Phi+\sum_{\alpha} \frac{\Phi(\vec{k})}{\lambda_{D \alpha}}\left\{1-\Gamma_{0}\left(k_{\perp}^{2} \rho_{\alpha}^{2}\right)\right\} \\
& =4 \pi \sum_{\alpha}\left[2 \pi q_{\alpha} \int_{-\infty}^{\infty} \delta F_{\alpha}(\vec{k}) J_{0}\left(k_{\perp} \rho_{\alpha}\right) e^{i \vec{k} \cdot \vec{X}} m_{\alpha}^{2} B_{0} d \nu_{\|} d \mu\right]
\end{aligned}
$$

Where $\delta F_{\alpha}=F-F_{M \alpha}$ with $F_{M \alpha}$ being the local equilibrium Maxwellian distribution function of the particle species $\alpha$. $\Gamma_{0}\left(k_{\perp}^{2} \rho_{\alpha}^{2}\right)=I_{0}\left(k_{\perp}^{2} \rho_{\alpha}^{2}\right) \exp \left(-k_{\perp}^{2} \rho_{\alpha}^{2}\right), \quad I_{0}\left(k_{\perp}^{2} \rho_{\alpha}^{2}\right)$ and $J_{0}\left(k_{\perp} \rho_{\alpha}\right)$ are the modified and the first kind of Bessel functions. $\rho_{\alpha}$ and $\lambda_{D \alpha}$ are the Larmor radius and Debye lengths of the particle species $\alpha$, respectively. In this work, $\alpha=i, e$ are taken. This gyrokinetic model can be generally employed for first principle multi-scale ITG+ETG simulation if $\delta F_{\alpha}$ is directly solved for ions and electrons on equal foot. Starting from it, reduced version for the individual ITG or ETG turbulence may be derived with an assumption of adiabatic

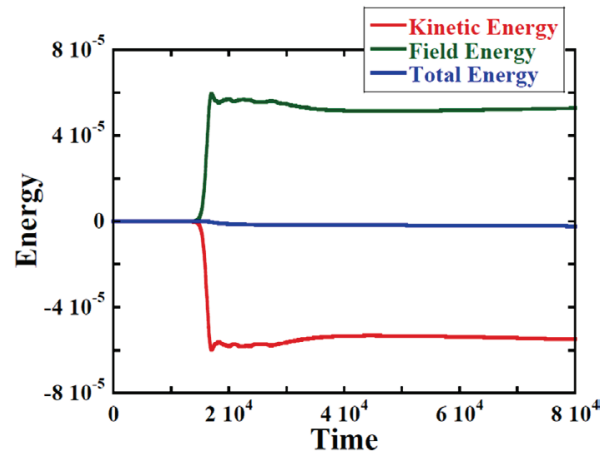

Fig. 7 Time history of kinetic, field and total energy. The time is normalized by $v_{t i} / \rho_{t i}$ with ion Larmor radius $\rho_{t i}$.

component response. ${ }^{7,25)}$ Furthermore, a drift-kinetic model can be obtained by assuming small FLR effect. In the following, the drift-kinetic ITG turbulence is taken as an example for the sake of simplicity to develop a Vlasov code with advanced CFD scheme. The code application to the multi-scale ITG+ ETG simulation is straightforward with high performance parallelization. The corresponding normalized 4D (3D in real space and 1D in velocity space) drift-kinetic Vlasov and Poisson equations in slab geometry is given as, ${ }^{26}$ )

$$
\begin{aligned}
& \frac{\partial f_{i}}{\partial t}-\frac{\partial \Phi}{\partial y} \frac{\partial f_{i}}{\partial x}+\frac{\partial \Phi}{\partial x} \frac{\partial f_{i}}{\partial y}+v_{\|} \frac{\partial f_{i}}{\partial z}-\frac{\partial \Phi}{\partial z} \frac{\partial f_{i}}{\partial v_{\|}}=0 \\
& -\nabla_{\perp}^{2} \Phi+\tau\left(\Phi-<\Phi>_{y z}\right)=\int f_{i} d u_{\|}-1
\end{aligned}
$$

Here $f_{i}=2 \pi \int F_{i} m_{i}^{2} B_{0} d \mu, \tau=T_{i} / T_{e}$. The adiabatic electron response is obtained through averaging the ES potential on the $y-z$ plane $<\Phi>_{y z}=\iint_{y z} \Phi d y d z / L_{y} L_{z}$.

A conservative form of the Interpolated Differential Operator (IDO-CF) scheme ${ }^{27}$ is applied to solve 4D gyrokinetic Vlasov-Poisson equation system incorporating with the Morinishi scheme, ${ }^{28)}$ in which not only the distribution function $f_{i}$ but also its integrated values in $v_{/ /}$-direction between girds, i.e., $\rho=\int_{v_{j}}^{v_{j+1}} f_{i} d v_{\|}, \quad u=\int_{v_{j}}^{v_{j+1}} v_{\|} f_{i} d v_{\|}, \quad e=\int_{v_{j}}^{v_{j+1}} v_{\|}^{2} f_{i} d v_{\|}$ are traced. ${ }^{27)}$ These integrated values are directly related to the velocity moments by summing up each component in $v_{/ /}$-direction. In the code, the spatial derivative is discretized by the Morinishi scheme, whereas the derivative to $v_{/ /}$ is done by the IDO-CF one, which ensures the rigorous conservation of mass, momentum and energy. Figure 7 plots energy evolution as an example for benchmark. Here the density and electron temperature are assumed to be homogeneous, whereas the initial ion temperature profile is given as $T_{i 0}(x)=1-\left(L_{x} / 2 \pi L_{T 0}\right) \cos \left(2 \pi x / L_{x}\right)$ with $L_{T 0}=37$. A shearless slab geometry is employed with the system size of $L_{x}=2 L_{y}=64$ and $L_{z}=8,000$ in real space, and $L_{v}=10$ in velocity space. Periodic boundary conditions are used in all the directions in real space $(x, y, z)$ and grid number $\left(N_{x}, N_{y}, N_{z}, N_{v \|}\right)=(256,128,32,128) \quad$ is typically chosen. About $3.86 \%$ of the relative total energy error after 16,000 time step is observed, showing the code validity with excel- 
lent energy conservation for long time behaviors.

The multi-scale gyrokinetic ITG+ETG micro-turbulence simulation requires a tremendous amount of computer resources. The code performance closely depends on the parallelization efficiency using several tens of thousands of CPUs. Hence the gyrokinetic code is further advanced by parallelizing the finite difference solver and the FFT solver with 1D ( $x$ or $k_{x}$ ) or 2D domain decomposition $(x-y$ or $k_{x}-k_{y}$ ). Figure 8(a) shows the speed up rate of total parallelization performance. In the case of 2D decomposition, the total speed up rate with $1,024 \mathrm{CPU}$ becomes $47.8 \%$, whereas that with 512 CPU is still $11.6 \%$ in the case of $1 \mathrm{D}$ decomposition. The Vlasov solver and the Poisson solver have the same high efficiency in both 1D and 2D parallelization decomposition. The difference mainly originates from the data communication as shown in Fig. 8(b). In 1D decomposition, the data communication for Vlasov solver costs CPU time about four times longer than that in 2D case by using MPI_SENDRECV. Meanwhile, the CPU time for data communication in Poisson solver using MPI_ALLTOALL takes about ten times longer. These comparisons show that 2D decomposition is much efficient for large-scale Vlasov simulation.

As a practical step towards the multi-scale gyrokinetic ITG+ETG micro-turbulence simulation, a sheared slab geometry in tokamak configuration is employed first to simulate the underlying nonlinear interaction between ion-scale and electron-scale turbulent dynamics. As an important physical process related to thermodynamics and the corresponding micro-scale phase space fluctuations, the entropy dynamics in gyrokinetic ITG turbulence has been investigated based on a global simulation. ${ }^{26)}$ It is found that the entropy convection plays an important role in the relaxation dynamics dominated by the avalanche process. A self-organized relaxed state is established, in which short-wavelength temperature corrugation, i.e., zonal pressure, is regulated by zonal flow shear. The gyrokinetic Vlasov code is being advanced to simulate full kinetic ion and electron dynamics on equal foot in the slab geometry and also to approach to a realistic tokamak toroidal configuration.

\section{Summary}

In this work, two numerical approaches based on both gyrofluid and gyrokinetic models are proposed to explore the cross-scale problems in MCF plasma involving the MHD island dynamics and micro-turbulence as a practical step towards a full-scale turbulence simulation. The attention is focused on the nonlinear interaction mechanism between different modes with different scales and EM features. At first, the gyrofluid simulations have been performed to clarify the energy-exchange mechanism between ES and EM fluctuations in multi-scale MHD and ion-scale micro- turbulence. It is found that a magnetic island seesaw oscillation and a short wavelength ion-scale drift wave appear in multi-scale turbulence simulations with different equilibrium magnetic field. The island seesaw mechanism is identified as a cross-scale dynamo generation by the micro-turbulence with an elucidation of a minimal modeling analysis. Mean-
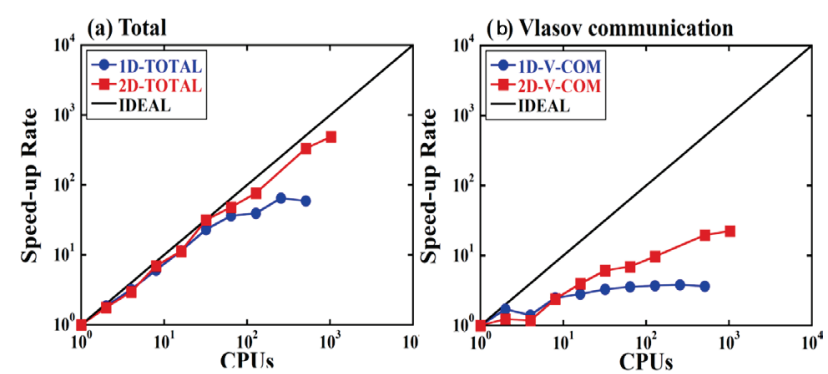

Fig. 8 Speed-up rate of MPI-parallelization in the case of 1D decomposition (blue line) and 2D decomposition (red line). Ideal rate (black line) are also shown as a reference.

while the short wavelength instability is induced by a wide magnetic island under the temperature island collapse. Secondly, a strategic plan to start a multi-scale gyrokinetic micro-turbulence simulation is introduced aiming at directly solving both adiabatic kinetic ion and electron responses for the long time plasma behaviors. The Vlasov code using IDO-CF scheme is developed with high parallelization performance through the decomposition in 2D real or spectral domain. The code benchmark and initial simulations of the global ITG turbulence show the promising potential for large-scale simulation of the plasma turbulence and transport.

\section{Acknowledgment}

This work was supported by the Grant-in-Aid from the JSPS with Grant No. 19560828 and No. 21340171. It was also partially supported by the JSPS-CAS (Japan-China) Core University Program on Plasma and Nuclear Fusion and by the JSPS Asian CORE program on Plasma and Nuclear Fusion.

\section{References}

1) IAEA, http://rpop.iaea.org

2) E. J. Doyle et al., Nucl. Fusion, 47, S18 (2007).

3) A. J. Brizard, T. S. Hahm, Rev. Mod. Phys., 79, 421 (2007).

4) X. Garbet et al., Nucl. Fusion, 50, 043002 (2010).

5) J. Wesson, Tokamaks, 2nd Ed.,Clarendon Press, Oxford (1997).

6) J. W. Connor et al., Phys. Rev. Lett., 40, 396 (1978).

7) Z. Lin et al., Science, 281, 1835 (1998).

8) F. Zonca et al., Phys. Plasmas, 6, 1917 (1999).

9) G. W. Hammett et al., Phys. Rev. Lett., 64, 3019 (1990).

10) W. Dorland et al., Phys. Fluids, B5, 812 (1993).

11) W. Horton et al., Phys. Fluids, 31, 2971 (1988).

12) J. Li et al., Phys. Plasmas, 11, 1439 (2004).

13) W. Horton, Rev. Mod. Phys., 71, 735 (1999).

14) J. Li et al., Nucl. Fusion, 49, 095007 (2009).

15) J. Li et al., Phys. Plasmas, 5, 959 (1998).

16) P. H. Rutherford, Phys. Fluids, 16, 1903 (1973).

17) N. F. Loureiro et al., Phys. Rev. Lett., 95, 235003 (2005).

18) R. Fitzpatrick, Nucl. Fusion, 33, 1049 (1993).

19) J. Li et al., Plasma Fusion Res., 5, 031 (2010).

20) Z. Chang et al., Nucl. Fusion, 34, 1309 (1994).

21) A. Isayama et al., Plasma Phys. Control. Fusion, 41, 35 (1999). 
22) Z. X. Wang et al., Phys. Plasmas, 16, 060703 (2009).

23) J. Candy et al., Plasma Phys. Control. Fusion, 49, 1209 (2007).

24) T. Gorler et al., Phys. Rev. Lett., 100, 185002 (2008).
25) Y. Idomura et al., Nucl. Fusion, 45, 1571 (2005).

26) K. Imadera et al., Plasma Fusion Res., 5, 019 (2010).

27) Y. Imai et al., J. Comput. Phys., 227, 2263 (2008).

28) Y. Morinishi et al., J. Comput. Phys., 143, 90 (1998). 